\title{
Reaction time as a measure of summation of warmth*
}

\author{
WILLIAM P. BANKS $\dagger$ \\ Pomona College, Claremont, California 91711 \\ and \\ The John B. Pierce Foundation Laboratory, New Haven, Connecticut 06519
}

\begin{abstract}
Reaction time (RT) to radiant heat applied to the forehead and to the back was found to decrease monotonically as a function of two variables: intensity (flux density) and areal extent of stimulation. Intensity and area can thus be traded one for the other to preserve a constant RT. It has previously been shown that intensity and area can be traded to preserve a constant degree of apparent warmth, as determined by magnitude estimation. $\mathrm{RT}$ and magnitude estimation therefore agree in showing that the warmth sense displays spatial summation, and both show that summation is very great. They also agree in showing that the forehead's sensitivity greatly exceeds the back's, and the exact trading rules revealed by magnitude estimation and RT agree well at low and moderate intensities.
\end{abstract}

Stimulus intensity affects reaction time (RT) in many sensory modalities (Piéron, 1914, 1952). As intensity increases, RT decreases. With a notable exception (Wright, 1951), studies of warmth have concerned only one of the two main variables that determine the magnitude of perceived warmth, namely, stimulus intensity. The areal extent of skin stimulated is, at least at threshold, no less important than intensity in determining the felt strength of a warm stimulus. The intensity of a stimulus can be systematically decreased as area is increased and maintain a constant level of perceived warmth; and, as a corollary, changes in areal extent of stimulation will, with a constant stimulus intensity, be perceived as changes in warmth. This trading relationship between area and intensity is termed spatial summation of warmth. The present study utilizes RT as a measure of spatial summation and shows that RT varies with areal extent as well as intensity of stimulation and can measure summation.

Threshold measurements have predominated in previous investigations of spatial summation of warmth. These have shown that summation can extend over large areas of skin (Hardy \& Oppel, 1937) and that summation is nearly perfect at threshold; that is, within limits, area and intensity can be traded equally to

*This research was supported by Grant AFOSR-70-1950 from the Air Force Office of Scientific Research. A Pomona College Summer Research Fellowship provided support for the author while conducting this research at The John B. Pierce Foundation Laboratory. The author is grateful to J. C. Stevens for his original suggestion that reaction time might demonstrate summation of warmth and for his detailed critical reading of several drafts of the paper. The author also thanks J. D. Hardy for a critical reading of the paper. L. E. Marks also made valuable comments on several drafts of the manuscript. Portions of this research were reported at meetings of the Psychonomic Society, San Antonio, Texas, 1970, and at the International Symposium on Environmental Physiology, Dublin, Ireland. 1971.

†Requests for reprints should be sent to William P. Banks, Department of Psychology, Pomona College. Claremont. California 91711. preserve the threshold of feeling (Hardy \& Oppel, 1937; Kenshalo, Decker, \& Hamilton, 1967). In contrast, the threshold of pain exhibits little or no summation (Greene \& Hardy, 1958). At an intermediate intensity (the threshold of "heat"), areal summation does take place, but it is not as complete as at the threshold of feeling (Herget \& Hardy, 1942). Stevens and Marks (1971) have used the technique of magnitude estimation to study summation of warmth at a large number of areal extents and intensities of stimulation. Their results show that summation is best near threshold and that it systematically decreases with higher levels of warmth until painful levels are reached, where summation is negligible.

It has been argued that summation of warmth may result from a failure of the $S$ to distinguish the areal size of the stimulus from its apparent intensity (Baron \& Bentley, 1921; Geblewicz, 1938). By this argument, Ss report that larger stimuli feel warmer than smaller ones when they really mean to say they are simply larger. Since RT requires no estimation process that could be distorted by perceptual (or semantic) confusions, summation effects revealed by RT can confidently be attributed to sensory processes. Although Wright (1951) has demonstrated spatial summation with a RT technique, he did not present the reciprocity contours that would allow comparisons between his results and what is now known about summation at supraliminal levels.

In the present experiments, Wright's procedure of letting the S's response terminate the stimulus exposure was followed. The advantage of using this procedure is that very weak stimuli can be used, and summation can be studied at levels barely above absolute threshold. The standard procedure for studying the RT-intensity relationship is, however, to present the stimulus for a brief duration. The present RT measurements are therefore not directly interpretable as classical psychophysical RTs. Although the practice of letting the $S$ 's response terminate the stimulus introduces a 
confounding between stimulus strength and duration, there is no way that this confounding could artifactually create an apparent trading relationship between areal extent and intensity of stimulation where none existed. The mathematical form of the trading relation might, however, be different with fixed-duration pulses.

\section{METHOD}

Reaction times to radiant stimulation for various combinations of area and intensity were obtained for the back and forehead. The region stimulated was painted with India ink to insure uniform spectral absorption. All experiments were conducted in an air-conditioned room maintained at $20^{\circ} \mathrm{C}$ and $40 \%$ relative humidity. At the start of each session, the $S$ adapted to the conditions in the room for at least $10 \mathrm{~min}$.

\section{Experiments 1 and 2: Forehead}

The source of radiant heat was a $1,000-\mathrm{W}$ incandescent projection lamp housed in a metal chamber with an aperture at one side through which a fairly uniform beam of radiant energy was emitted. A variable transformer controlled the voltage across the lamp and thereby determined the stimulus intensity. A Hardy radiometer (Hardy, Wolff, \& Goodell, 1952) was used to calibrate the field and determine its uniformity. Over the period of the experiments, the output of the lamp remained constant within $1.5 \%$ of the mean calibration curve, and the denşity of the field at the S's forehead remained uniform within $\pm 2 \%$ of the mean value over most of its extent.

The $S$ sat in a comfortable position behind a cork-backed aluminum insulating screen through which the beam was cast on his forehead. The areal extent of stimulation was determined by cork-backed masks inserted in a slot in the screen between the $S$ 's forehead and the lamp. A shutter operated by a solenoid blocked the path of the radiant heat close to the lamp, and the $S$ controlled the shutter with a microswitch held in his hand. When the intensity and area for a given trial had been set, the $E$ gave a ready signal and the $S$ depressed the microswitch, opening the shutter. The $\mathbf{S}$ was instructed to release the switch as soon as he was sure he felt warmth on his forehead. For as long as the switch was depressed, a decade counter accumulated pulses from a $10,000-\mathrm{Hz}$ signal. The timing system was accurate to at least $0.2 \%$, but it overestimated RT by about $25 \mathrm{msec}$ because of microswitch and shutter lags.

Experiments 1 and 2 were identical except for the particular combinations of area and intensity used (Table 1 gives these combinations). The two experiments were performed in alternate experimental sessions, and the $S$ was never aware of which experimental series was being presented, or even that there were two such series.

\section{Experiment 3: Back}

Four General Electric $\mathrm{T}-3$ quartz lamps mounted in parabolic-cylindrical reflectors provided the radiant heat. The $S$ sat blindfolded in a chair with a $324-\mathrm{cm}^{2}$ circular aperture in the backrest approximately at the midthoracic level. Intensity was controlled by varying the voltage across the lamps, and areal extent was controlled by masks with circular apertures placed over the backrest. Table 1 gives the combinations of areas and intensities used. The field was calibrated and checked for uniformity with a Hardy radiometer. There was no measurable drift from the mean calibration curve over the course of the experiment, and the areal density of flux was uniform within $\pm 2 \%$ in the plane of stimulation.

Affixed to the rear of the S's chair, between the opening and the heat lamps, was a shutter constructed from a roller-type windowshade. The shade was covered with reflective foil, and when reieased by a solenoid latch it rolled up quickly to expose the back. After setting the intensity and area for a given trial. the $E$ removed a protective heat shield from the lamps, warning the $S$ that a trial was about to begin. Then he threw a switch, which released the shutter, exposing the S's back to the radiant heat, and which simultaneously started a Standard Electric centisecond timer. The $\mathrm{S}$ held a microswitch in his hand and was instructed to lift a finger from it as soon as he was sure he felt warmth on his back. Lifting the finger stopped the timer. Because the shutter moved across the backrest from the bottom to the top, the larger areas stimulated began to be exposed sooner than the smaller ones but took longer to be completely exposed. The total difference between the largest and smallest areas is, however, only $20 \mathrm{msec}$, an amount completely dwarfed by the obtained difference in RTs for these areas. All RTs were overestimated by $50 \mathrm{msec}$ because of microswitch and shutter delays. Random errors in the timing system were less than $\pm 0.5 \%$.

\section{Procedure: Experiments 1, 2, and 3}

After the $S$ had acclimated to the experimental room and before experimental trials commenced, some warm-up RT trials, using various areas and intensities, were administered. Warm-up lasted for 4 trials on the back and from 10 to 20 or more on the forehead. The experimental session, including short rest periods, continued for approximately $1 \mathrm{~h}$ after warm-up.

Every session in each of the three experiments contained, in equal number, every area-intensity combination used in that experiment. If different blocks of stimuli had been presented in different sessions, shifts in response criterion could have washed out stimulus intensity effects (cf. Grice \& Hunter, 1964; Murray, 1970). In Experiment 1, a large number of area-intensity combinations were presented for forehead RTs. Experiment 2 had a smaller number of combinations but more observations per combination. Experiment 3 examined the back. In all three experiments, the combinations of area and intensity were presented in irregular order except that extreme contrasts in intensity were not presented successively. In all sessions, the interstimulus interval was at least $10 \mathrm{sec}$ and the mean interval was about $15 \mathrm{sec}$ for the forehead and $40 \mathrm{sec}$ for the back. Catch trials were given in all sessions at a ratio to data trials of 1:10 for the forehead and 1:15 for the back.

\section{Subjects}

There were two paid Ss, both males in their 20s. One (T.B.) served in 12 90-min sessions of pilot work and the other (D.A.) in 9 such sessions. Two other paid Ss served in only 2 sessions each. One became unavailable after the second session, and the other was dismissed because of excessive variability of RT. Three members of the laboratory staff also contributed to pilot work. The limited data from these five Ss show essentially the same features as do T.B.'s and D.A.'s.

\section{RESULTS AND DISCUSSION}

Table 1 presents the geometric mean RTs obtained in the three experiments. The geometric mean was chosen over the arithmetic mean because the data are positively skewed, but because the variance of RTs was relatively small, the geometric means averaged only about $1 \%$ smaller than the arithmetic means. All stimuli were well above threshold and none was missed. Neither $\mathrm{S}$ gave a false positive response on any of the approximately 300 catch trials he had.

Table 1 shows that increases in both intensity and 
areal extent reduce the RT. For any given extent, RT falls off rapidly with increases in intensity. Also, for any given intensity, RT falls off rapidly with areal increases.

Experiment 2 combined intensity and area orthogonally and lends itself easily to an analysis of variance. The logarithms of the RTs were used in the analysis, and area, intensity, Ss, and sessions were the variables in a four-way orthogonal design. Main effects of and interactions among area, intensity, and session were tested against the appropriate interaction with Ss. The $S$ variable and interactions involving it were tested against the mean within-cell error. Both area, with an $F(2,2)$ of $1,045.7$, and intensity, with an $F(3.3)$ of 39.6 , were significant at the $p<.01$ level. The Ss, with an $F(1,1056)$ of 840.9 , also differed significantly. The sessions did not differ reliably, $F(3,3)=2.00$. Four interactions were significant at the .01 level: Intensity by Area, $F(6,6)=37.9$; Ss by Sessions, $F(3.1056)=$ 18.4; Ss by Intensity, $F(3,1056)=9.68$; and $S s$ by Sessions by Area, $F(6,1056)=7.54$.

These results emphasize what is clear in Table 1. Area and intensity both determine the RT, with area probably more important a variable than intensity. The interaction between area and intensity is to be expected from the fact that RT decreases with increases in both variables but appears to approach a limiting minimum value as they increase. The significant overall difference between the Ss is also obvious in Table 1. The lack of a significant effect of sessions is to be expected from the fact that the $S s$ had had extensive practice before experimental trials began. The significant Ss by Intensity interaction reflects the fact that D.A. showed a slightly greater intensity effect than did T.B., but the other two significant interactions involving $S s$ have no simple explanation. However, they represent fairly small mean fluctuations and should not complicate the interpretation of the results.

\section{Relationship between Response Speed and Magnitude Estimation of Warmth}

In vision, the $R T$ vs intensity function is predictable from the psychophysical brightness function (Vaughan, Costa, \& Gilden, 1966). Both response speed and judged brightness increase as the $1 / 3$ power of luminance. Do response speed and judged warmth both rise with the same power of intensity of radiant heat? This can be answered by converting the RTs to speed and comparing the speed functions with Stevens and Marks's (1971) magnitude scales of warmth.

In Figs. 1 and 2, the RTs from Experiments 1 and 3 are converted to speed and plotted as a function of intensity, with area as the parameter. (The speed plots for Experiment 2 are much the same as those for Experiment 1 and were omitted because they add little information.) Speed equals $1 /(R T-K)$. where $K$ is 3 constant representing the nonintensity-dependent component of the RT. $K$ is assumed to measure the
Table 1

Geometric Mean RTs for Ss DA and TB in Experiments 1, 2, and 3*

Experiment 1 (Forehead)

\begin{tabular}{|c|c|c|c|}
\hline \multirow{2}{*}{$\begin{array}{l}\text { Area } \\
\mathrm{cm}^{2}\end{array}$} & \multirow{2}{*}{$\begin{array}{l}\text { Intensity } \\
\mathrm{mW} / \mathrm{cm}^{2}\end{array}$} & \multicolumn{2}{|c|}{$\begin{array}{c}\text { Reaction Time } \\
\text { (Msec) }\end{array}$} \\
\hline & & DA & TB \\
\hline 3.65 & 133 & $2994^{*}$ & 2664 \\
\hline 3.65 & 163 & $1922^{\circ}$ & 2423 \\
\hline 3.65 & 210 & $1469^{*}$ & 1766 \\
\hline 3.65 & 284 & $1268^{\circ}$ & 1516 \\
\hline 3.65 & 359 & $1044^{*}$ & 1090 \\
\hline 5.72 & 105 & 2671 & 1875 \\
\hline 5.72 & 163 & 1488 & 1245 \\
\hline 5.72 & 247 & 1027 & 1038 \\
\hline 5.72 & 341 & 868 & 749 \\
\hline 9.65 & 74 & 1991 & 1351 \\
\hline 9.65 & 133 & 1284 & 1136 \\
\hline 9.65 & 210 & 942 & 775 \\
\hline 9.65 & 341 & 741 & 559 \\
\hline 14.5 & 74 & 1124 & 935 \\
\hline 14.5 & 133 & 999 & 763 \\
\hline 14.5 & 210 & 782 & 527 \\
\hline 14.5 & 341 & 640 & 493 \\
\hline 21.8 & 49 & 1366 & 869 \\
\hline 21.8 & 74 & 975 & 802 \\
\hline 21.8 & 133 & 791 & 562 \\
\hline 21.8 & 210 & 704 & 468 \\
\hline 21.8 & 341 & 623 & 417 \\
\hline
\end{tabular}

Experiment 2 (Forehead)

\begin{tabular}{|c|c|c|c|}
\hline $\begin{array}{r}9.65 \\
9.65 \\
9.65 \\
9.65 \\
21.8 \\
21.8 \\
21.8 \\
21.8 \\
39.0 \\
39.0 \\
39.0 \\
39.0 \\
\end{array}$ & $\begin{array}{l}105 \\
163 \\
247 \\
341 \\
105 \\
163 \\
247 \\
341 \\
105 \\
163 \\
247 \\
341 \\
\end{array}$ & $\begin{array}{r}1809 \\
1264 \\
960 \\
796 \\
1008 \\
793 \\
676 \\
616 \\
674 \\
601 \\
546 \\
506 \\
\end{array}$ & $\begin{array}{r}1197 \\
997 \\
742 \\
624 \\
723 \\
583 \\
527 \\
491 \\
453 \\
419 \\
414 \\
396 \\
\end{array}$ \\
\hline \multicolumn{4}{|c|}{ Experiment 3 (Back) } \\
\hline $\begin{array}{r}50 \\
50 \\
50 \\
127 \\
127 \\
127 \\
127 \\
324 \\
324 \\
324 \\
324\end{array}$ & $\begin{array}{r}110 \\
175 \\
270 \\
76 \\
110 \\
175 \\
270 \\
36 \\
54 \\
110 \\
270\end{array}$ & $\begin{array}{r}5448 \\
3017 \\
1813 \\
4055 \\
2649 \\
1442 \\
1105 \\
3226 \\
2652 \\
1331 \\
825\end{array}$ & $\begin{array}{r}2712 \\
1934 \\
1473 \\
1731 \\
1360 \\
1082 \\
1018 \\
1422 \\
1187 \\
964 \\
768\end{array}$ \\
\hline
\end{tabular}

Note-There were 24 obsenations per point in Experiment 1 (except $* 16$ obsenations). 48 per point in Experiment 2. and 12 per point for $D A$ and 24 for $T B$ in Experiment 3.

fixed lags in the response (e.g.. "muscle time," apparatus $\mathrm{lag}$ ), it is assumed to add equally to all RTs regardless of stimulus intensity. and it is the astmptotic limit the RT approaches as stimulus intensity increases (cf.. Piśron. 1914. 1919. 1952). The $K$ chosen for converting the 


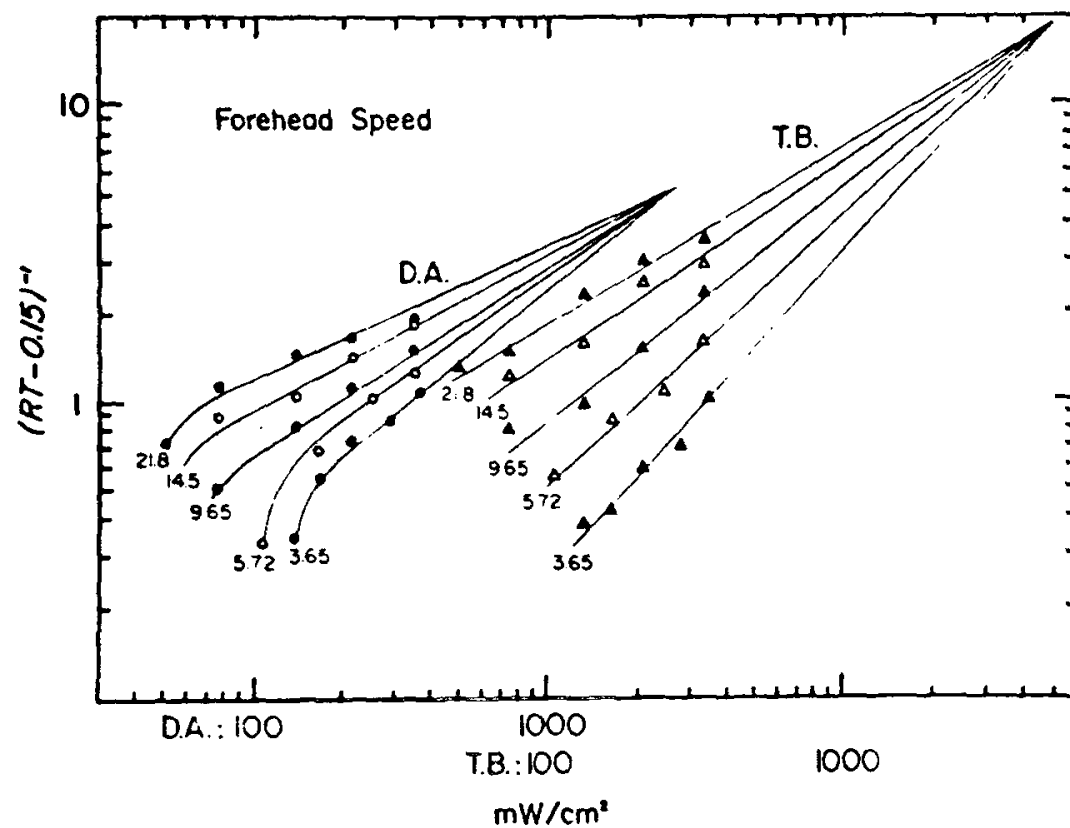

Fig. 1. Speed of response as a function of irradiance $\left(\mathrm{mW} / \mathrm{cm}^{2}\right)$ for five areal extents $\left(\mathrm{cm}^{2}\right)$ of the forehead. Results are from Experiment 1, and $S s$ are D.A. and T.B. Log-log coordinates.

RTs of the forehead (Experiment 1) was $150 \mathrm{msec}$, and that for the back (Experiment 3) was $200 \mathrm{msec}$.

\section{Estimating the Size of $\mathrm{K}$}

These values of $K$ are somewhat arbitrary and were settled upon after a number of attempts at estimating $\mathrm{K}$ were unsuccessful. The best way to estimate $K$ is to assess the asymptotic value of the RT. Unfortunately, none of the RT functions of the three experiments become flat enough within the range of intensity used to allow the asymptote to be determined accurately. (After these experiments were finished and difficulty in estimating $K$ was experienced, an apparatus capable of delivering $1,700 \mathrm{~mW} / \mathrm{cm}^{2}$ to the forehead was used in a
RT experiment with a new $S$. The experiment was not completed because above $1,200 \mathrm{~mW} / \mathrm{cm}^{2}$ the response was to pain, not warmth, and the RT function still did not seem close to an asymptote at $1,700 \mathrm{~mW} / \mathrm{cm}^{2}$.)

A second approach, used by Wright (1951) and discussed by Piéron (1952, pp. 345ff), is to base $\mathrm{K}$ on neural conduction and muscular response times. but only peripheral constant latencies can be estimated in this way and $\mathrm{K}$ is likely to be underestimated. Wright's physiological estimates give $\mathrm{Ks}$ of about $70 \mathrm{msec}$. Lsing $70 \mathrm{msec}$ as $\mathrm{K}$ would leave more curvature in the speed functions of Figs. 1 and 2, but they would still converge at nearly the same point.

A third approach is to estimate $\mathrm{K}$ as a parameter in a psychophysical equation fitted to the RT-intensity

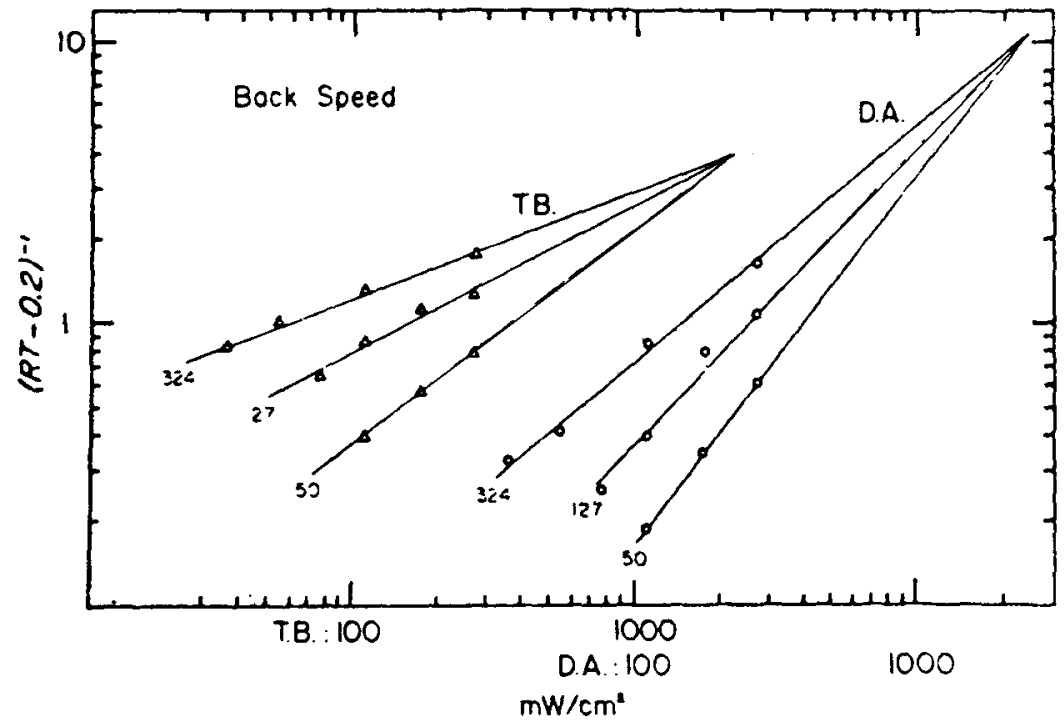

Fig. 2. Speed of response as a function of irradiance $\left(\mathrm{mW} / \mathrm{cm}^{2}\right)$ for three areal extents $\left(\mathrm{cm}^{2}\right)$ of the back. Results are from Experiment 3, and $S s$ are D.A. and T.B. Log-log coordinates. 
functions. A fairly exhaustive series of attempts to estimate $K$ in this way was performed on an IBM 360 computer with various programs to fit the data with the different equations considered. Power, logarithmic, exponential, and linear functions were used, in separate attempts, to relate both response speed and RT either to $\mathrm{mW} / \mathrm{cm}^{2}$ or to terminal surface skin temperature (skin temperature at time of response). The $r^{2}$ criterion of goodness of fit was used, and it was expected that at least one of the equations would converge on a best-fitting value of $K$. Unfortunately, this was not the case. Most of the equations gave low values of $r^{2}$ overall and did not show peaks in the $r^{2}$ function where the best $\mathrm{K}$ might be located. Often, the best $\mathrm{K}$ was either negative or equal to the shortest $\mathrm{RT}$. One reassuring fact as far as the relationship between $\mathrm{RT}$ and magnitude estimation is concerned is that the power function yielded the largest $r^{2} s$ overall. However, even the power function failed to give consistent estimates of $\mathrm{K}$. It was finally decided, after these attempts to estimate $\mathrm{K}$ turned out to be inconclusive, that the power functions relating response speed $10 \mathrm{~mW} / \mathrm{cm}^{2}$, as shown in Figs. 1 and 2 , give the best available approximation to the empirical relationship. Although the Ks of 150 and $200 \mathrm{msec}$ in these figures were chosen only on the basis of overall visual fit, they yield an excellent fit in the power function. For D.A., the average $r^{2}$ values for the areas used in Experiments 1, 2, and 3 are .956, .999, and .990 , respectively, and for T.B., they are $.965, .963$, and .977.

\section{Speed, Magnitude, and Trading Functions}

The prima facie agreement between the speed functions of Figs. 1 and 2 and Stevens and Marks's (1971) magnitude scales is good. Figure 3a shows the magnitude for warmth obtained in their experiment for various areal extents of the forehead. The speed and magnitude functions seem to exhibit the same form: progressive reduction in slope as area increases and convergence toward a common point. The two sets of functions differ in that the speed functions are flatter than the magnitude scales (having about half the slope at corresponding areas) and converge at a greater intensity.

Figure $3 b$ shows trading functions derived by Stevens and Marks from the data of Fig. 3a. These functions show combinations of intensity and area that yield the same judgment of warmth. They were obtained by making horizontal cuts across the functions of Fig. 3a and finding the intersection of these horizontal cuts with the fitted magnitude functions for each area. Figures 4 and 5 show equal speed contours derived from horizontal cuts through the speed functions of Figs. 1 and 2 , respectively. The equal speed and equal magnitude contours are in general agreement. For combinations of area and intensity eliciting either judgments of how intensity or slew RTs summation is complete. or nearly so: i, e. intertsity and areat can be
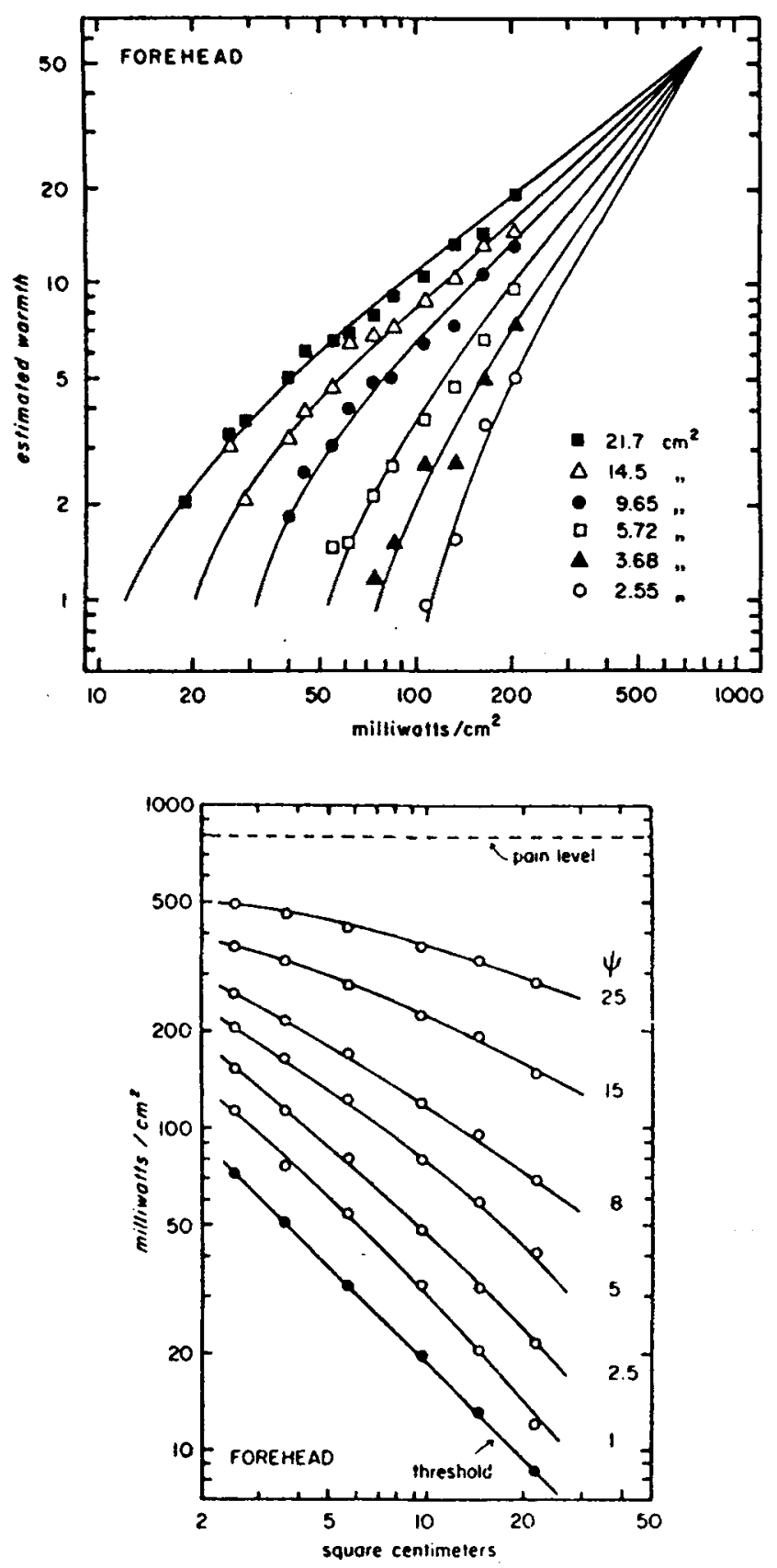

Fig. 3. (a) From Fig. 2 of Stevens and Marks (1971): estimates of warmth for six areas of the forehead: (b) from Fig. 6 of Stevens and Marks: equal-sensation contours derived from Fig. 3a. Log-log coordinates in both cases.

traded equally to preserve the same response speed or magnitude of warmth. With faster response speeds or more intense levels of reported warmth, areal summation becomes less and less important, as the progressive nattening of the trading relation indicates.

A further agrement helween the RT and masnitude estmation fintings is the relative unit areal sensitivity of

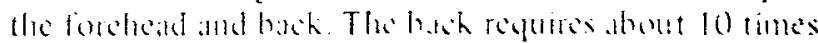




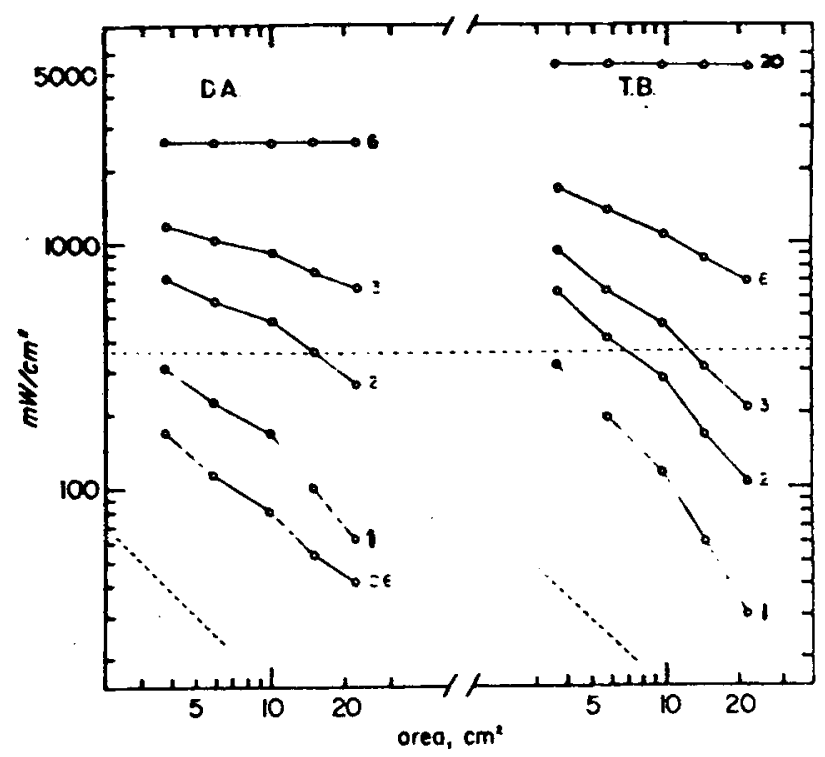

Fig. 4. The combinations of area $\left(\mathrm{cm}^{2}\right)$ and intensity $\left(\mathrm{mW} / \mathrm{cm}^{2}\right)$ that give a constant response speed. The various contours are derived from Fig. 1. The parameter is speed, as defined in that figure. The dashed horizontal line indicates the highest intensity at which data were collected. The Ss are D.A. and T.B. Log-log coordinates.

the areal extent of stimulation for a given intensity to elicit the same PT or the same judgment of magnitude of warmth.

There is a disagreement between the speed and magnitude area-intensity trading functions in the point at which areal summation ceases to operate. The magnitude functions predict that summation would

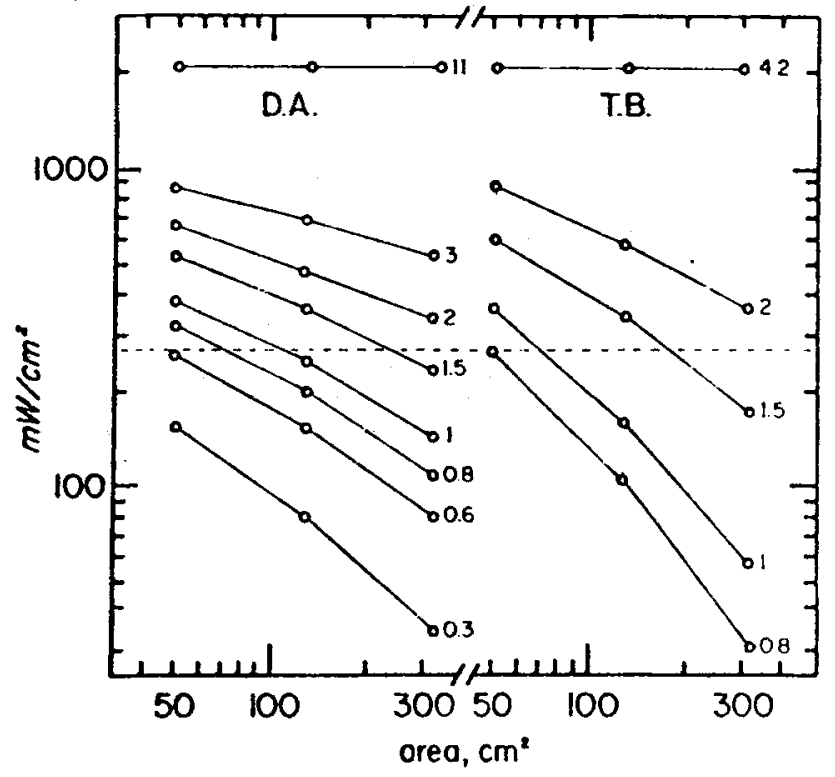

Fig. 5. Area-intensity trading relations for the hack, derived from Fig. 2 in the same way Fig. 4 was derived from Fig. 1. Log-log courdinates. cease at an intensity level of about $800 \mathrm{~mW} / \mathrm{cm}^{2}$. Since this level is one of painful stimulation and there is no spatial summation beyond the threshold of pain. it would be surprising if the trading relation for magnitude estimation did not become flat there. The specd functions, however, predict that areal summation would fail at much higher intensities: on the forehead at about $2,600 \mathrm{~mW} / \mathrm{cm}^{2}$ for D.A. and at $5,500 \mathrm{~mW} / \mathrm{cm}^{2}$ for T.B.: on the back at $2,100 \mathrm{~mW} / \mathrm{cm}^{2}$ for both Ss. It is possible that response speed could continue to increase and show summation beyond levels of intensity that would be painful if they were not terminated by the response. Stevens and Marks presented their stimuli for $3 \mathrm{sec}$ and noted that subjective intensity tended to build up during this period. In the brief period required for a response to $800 \mathrm{~mW} / \mathrm{cm}^{2}$, the sensation would probably not build up to a painful level and some summation could still be expected.

\section{CONCLUSIONS}

(1) The RT to radiant heat decreases with increases in areal extent of stimulation as well as intensity. Thus, areal summation of warmth at supraliminal levels can be shown with a technique that does not require judgment by the $S$.

(2) When RT is expressed as a power function of intensity, some relationships between the RTs and magnitude estimation scales of warmth are apparent. Both techniques show that areal summation is best near threshold, and both techniques also agree in showing that areal summation progressively decreases as intensity increases. The RT functions predict, however, that summation will fail at a much higher intensity than the magnitude functions predict. Both techniques show that the back is less sensitive than the forehead by about the same amount. It must be noted that some of the relationships between $\mathrm{RT}$ and magnitude estimation might be different if fixed-duration stimuli (rather than the present $S$-terminated stimuli) were used.

(3) Areal summation seems to be at least partly a central matter, because, for both the forehead and back. summation of warmth does not respect dermatomal boundaries.

\section{REFERENCES}

Baron, A., \& Bentley, M. The intensive summation of thermal sensations. Psychological Monographs, 1921, 30 (No. 3), 84-94.

Gcblewicz, L. La sommation spatiale des excitations thermiques. Annéc Psychologique, 1938, 39, 199-217.

Greene, 1. C., \& Hardy, J. D. Spatial summation of pain. Journal of Applicd Physiology, 1958, 13, 457-464.

Grice, G. R., \& Hunter, J. J. Stimulus intensity effects depend on the type of experimental design. Psychological Review, $1964,71,247-256$.

Hardy, J. D., \& Oppel, T. W. Studies in tempcrature sensation. III. The sensitivity of the body to heat and the spatial summation of the end-organ response. Journal of Clinical Investigation, 1937, 16, 533-540. 
Hardy, J. D., Wolff, H. G., \& Goodell, H. Pain sensations and reactions. Baltimore: Williams \& Wilkins, 1952.

Herget, C. M., \& Hardy, J. D. Temperature sensation: The spatial summation of heat. American Journal of Physiology, 1942, $135,426-429$.

Kenshalo, D. R., Decker, T., \& Hamilton, A. Spatial summation on the forehead, forearm, and back produced by radiant and conducted heat. Journal of Comparative \& Physiological Psychology, 1967.63, 510-515.

Murray, H. G. Stimulus intensity and reaction time: Evaluation of a decision-theory model. Journal of Experimental Psychology, 1970, 84, 383-391.

Piéron, H. Recherches sur les lois de variation des temps de latence sensorielle en fonction des intensités excitatrices. Année Psychologique, 1914, 20, 17-96.

Piéron, H. De la loi de variation des temps de latence en fonctions des intensités excitatrices par les sensations auditives. Comptes Rendus de la Societé de Biologie, 1919, $82,1116-1118$

Piéron, H. The sensations. London: Frederick Muller, 1952.

Stevens, J. C., \& Marks, L. E. Spatial summation and the dynamics of warmth sensation. Perception \& Psychophysics, 1971, 9, 391-398.

Vaughan, H. G., Jr., Costa, L. D., \& Gilden, L. The functional relation of visual evoked response and reaction time to stimulus intensity. Vision Research, 1966, 6, 645-656.

Wright, G. H. The latency of sensations of warmth due to radiation. Journal of Physiology, 1951, 112, 344-358.

(Received for publication July 31,1972 ; revision received December 8,1972 .) 